\section{Expression of stem cell factor and its receptor, c-kit, in human oral mesenchymal cells}

Gagari E, Rand MK, Tayari L, Vastardis H, Sharma P, Hauschka PV, Damoulis PD. Expression of stem cell factor and its receptor, c-kit, in human oral mesenchymal cells. Eur J Oral Sci 2006; 114: 409-415. (C) 2006 The Authors. Journal compilation (c) 2006 Eur J Oral Sci

Stem cell factor (SCF) is the pleiotropic ligand for the tyrosine kinase receptor, c-kit. Ligand and receptor are usually expressed in different cell types, and binding of SCF to c-kit promotes cell proliferation, differentiation, and recruitment of progenitor cells in various biologic systems. However, the localization of these two molecules in cells of the oral cavity has not been systematically examined. We investigated the expression of SCF and c-kit in human dental pulp (HDP) cells as well as in human gingival fibroblasts (HGF). Both alternatively spliced isoforms of SCF were detected (through reverse transcription-polymerase chain reaction) in RNA obtained from the two cell types. Western analysis established that both cell types express SCF and/or c-kit, whereas flow cytometry demonstrated distinct cell populations expressing only the ligand (SCF), only the receptor (c-kit), or co-expressing the two. HDP cultures showed higher soluble $\mathrm{SCF}$ (sSCF) production associated with faster cell growth, as compared with HGF cultures. In both cell types, however, sSCF levels appeared to increase as a result of in vitro aging and/or differentiation.
Eleni Gagari ${ }^{1}$, Matthew K. Rand ${ }^{2}$, Lili Tayari $^{3}$, Heleni Vastardis ${ }^{4}$, Parul Sharma ${ }^{5}$, Peter V. Hauschka ${ }^{5}$, Petros D. Damoulis ${ }^{6}$

${ }^{1}$ Tufts University School of Dental Medicine, Department of Oral and Maxillofacial Pathology, Boston, MA, USA; ${ }^{2}$ University of Michigan at Ann Arbor, Department of Oral and Maxillofacial Surgery, Ann Arbor, MI, USA; ${ }^{3}$ Private Practice, Boston, MA, USA; ${ }^{4}$ Tufts University School of Dental Medicine,

Department of Orthodontics, Boston, MA, USA; ${ }^{5}$ Children's Hospital Boston, Department of Orthopaedic Surgery, Boston, MA; ${ }^{6}$ Tufts University School of Dental Medicine, Department of Periodontology, Boston, MA, USA

Dr Petros D. Damoulis, Tufts University School of Dental Medicine, Department of Periodontology, DHS-6, One Kneeland Street, Boston, MA 02111, USA

Telefax: +1-617-6363401

E-mail: Petros.Damoulis@tufts.edu

Key words: c-kit; co-expression; dental pulp cells; gingival fibroblasts; stem cell factor

Accepted for publication May 2006
Stem cell factor (SCF, steel factor, kit ligand) is an important hematopoietic growth factor, which binds to and activates c-kit (CD117), a tyrosine kinase III receptor and product of the c-kit proto-oncogene (1). SCF exists in two alternatively spliced variants, differing in one exon (exon 6) (Fig. 1A) (2). The transcript that includes exon 6 encodes a transmembrane protein that can be cleaved proteolytically to produce a soluble molecule (sSCF) (3) that readily dimerizes (4). The transcript lacking exon 6 encodes a protein that translocates to the plasma membrane $(\mathrm{mSCF})$ that may also serve as a ligand for c-kit (Fig. 1B). sSCF can activate different signaling pathways from $\mathrm{mSCF}$, resulting in distinct biologic responses (5). SCF binding to c-kit induces receptor autophosphorylation and inititiation of a signaling cascade involving the phosphatidylinositol-3 (PI-3) Kinase/Akt and/or the Ras-mitogen-activated protein (MAP) kinase pathways (6). At least two variants of the c-kit receptor have been identified, differing only in four amino acids, which can be present or absent at the juxtamembrane portion of the extracellular domain of the receptor (6). Furthermore, c-kit can be cleaved by several proteases (7), and the resulting soluble c-kit receptor can have a strong inhibitory effect on SCF bioactivity (8).

Typically, SCF and c-kit are expressed by different cells in the same microenvironment. SCF is secreted by bone marrow stromal cells and supports hematopoietic cell survival and proliferation. This SCF role is partic- ularly important in mast cell growth and differentiation. A similar SCF/c-kit interplay has also been identified in other tissues, where cell recruitment and differentiation is necessary (2). Interestingly, several cell types have been shown to co-express SCF and c-kit, including human mast cells (9), mouse fetal oocytes (10), and human melanocytes (11). However, the majority of studies exploring the expression of the c-kit receptor and its ligand indicate a possible association of the co-expression with neoplastic transformation, primarily in epithelial tissues (11-13). Furthermore, ectopic expression of c-kit in NIH $3 \mathrm{~T} 3$ fibroblasts induces tumorigenesis (14). Recently, c-kit expression was demonstrated in human adult dental pulp cells that originate developmentally from the mesenchyme (15). Other cells of mesenchymal origin are known to express SCF, including fibroblasts from the oral mucosa (16). The goal of this study was to explore the pattern of expression of c-kit and SCF isoforms in fibroblast-like cells derived from human gingiva and dental pulp.

\section{Material and methods}

\section{Materials}

All tissue culture reagents were purchased from Mediatech/ Cellgro (Herndon, VA, USA), other chemicals from Sigma Diagnostics (St Louis, MO, USA), and plasticware from 
A

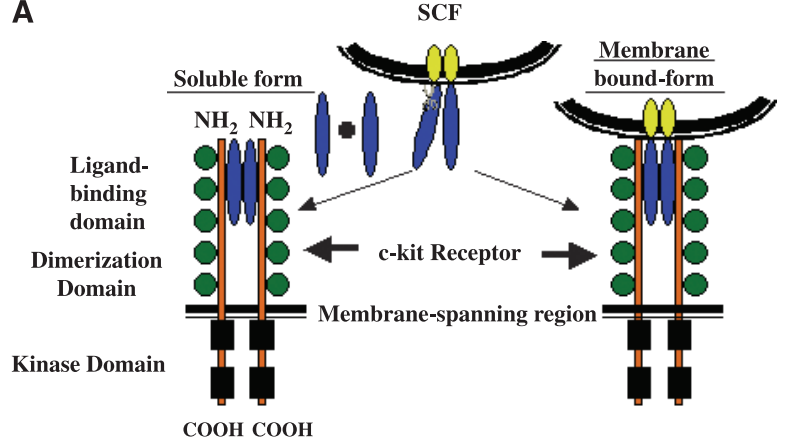

B

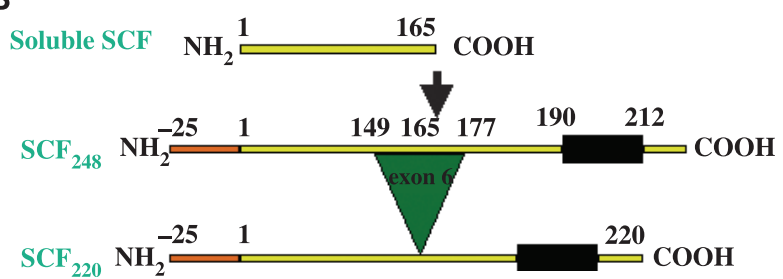

Fig. 1. Stem cell factor ( $\mathrm{SCF}$ ) isoforms and their interaction with c-kit. (A) SCF can exist either in a membrane-bound form or in a soluble form, the latter produced by proteolytic cleavage. Both forms can bind to c-kit, inducing its dimerization and intracellular signaling. The c-kit receptor contains five extracellular immunoglobulin-like domains (green circles), which are involved in ligand binding and receptor dimerization, as well as two intracellular tyrosine kinase domains (black squares), which initiate signal transduction. (B) Structural organization of the soluble and transmembrane forms of human SCF. The two transmembrane isoforms of SCF $\left(\mathrm{SCF}_{248}\right.$ and $\mathrm{SCF}_{220}$ ) differ in one exon (exon 6), which contains the primary proteolytic cleavage site, indicated by the arrow. Cleavage at this site generates the soluble form of SCF. The 25 -amino acid signal peptide sequence (orange) and the hydrophobic transmembrane domain (black) are also shown.

Corning/Costar (Corning, NY, USA), unless listed otherwise.

\section{Tissue culture}

Human gingival fibroblasts (HGF) were obtained from the American Type Culture Collection (HGF-1, CRL-2014; ATCC, Manassas, VA, USA) and maintained in Dulbecco's modified Eagle's medium, supplemented with $10 \%$ heatinactivated fetal bovine serum (FBS; Invitrogen, Carlsbad, CA, USA) and $4 \mathrm{mM}$ L-glutamine. HGF cells were used at passages 5-7. Human dental pulp (HDP) cells were obtained from intact extracted teeth, as previously described (17), using a protocol approved by the Tufts-New England Medical Center Institutional Review Board. At confluence, the cells were trypsinized and propagated until passage 2 . WS1 human skin fibroblasts and FSF human foreskin fibroblasts were a gift from Dr Theodora Danciu (Harvard School of Dental Medicine, Boston, MA, USA) and were cultured under the same conditions as HGF cells. The latter two are non-transformed cell lines with high proliferative potential, deriving from fetal (WS1) or newborn (FSF) tissue. Cells used for reverse transcription-polymerase chain reaction (RT-PCR) or western analysis were seeded in $10 \mathrm{~cm}$ dishes and cultured until confluent. For flow cytometry, cells were seeded in T75 flasks and cultured for
10-14 d. For enzyme-linked immunosorbent assay (ELISA), cells were seeded in six-well plates at a density of $10^{3} \mathrm{~cm}^{-2}$ and cultured for $17 \mathrm{~d}$. At each time point, cells were trypsinized, stained with Trypan blue, and counted in a hemacytometer.

\section{Alkaline phosphatase activity}

Adherent cells were lysed with $1 \%$ Triton-X-100 in Trisbuffered saline. Alkaline phosphatase (ALP) activity was measured in the cell lysates, as previously described (18). Enzymatic activity was expressed in pmoles para-nitrophenol $(\mathrm{pNP}) \mathrm{min}^{-1}$ per $\mathrm{mg}$ of protein.

\section{RT-PCR}

RNA was extracted from cells using the TOTALLY RNA kit, followed by DNAse digestion with DNA Free (Ambion, Austin, TX, USA). cDNA was synthesized using the Amplitaq Gold RNA PCR Core kit (PE Biosystems, Foster City, CA, USA). Approximately $1 \mu \mathrm{g}$ of RNA was reverse transcribed in a $20 \mu \mathrm{l}$ reaction volume. SCF amplification was performed using a pair of oligonucleotide primers (GGATGACCTTGTGGAGTGCG, sense; GCCCTTGTAAGACTTGGCTG, antisense), designed to include exon 6. Two amplified fragments are expected with this set of primers, one at $416 \mathrm{bp}$, reflecting the exon $6(+)$ transcript, and one at $330 \mathrm{bp}$, reflecting the exon $6(-)$ transcript (19). PCR amplifications were performed using reagents from the Amplitaq Gold RNA PCR Core kit (PE Biosystems) in a volume of $25 \mu \mathrm{l}$, containing $2.5 \mu \mathrm{l}$ of cDNA. Amplification of SCF cDNA was achieved by an initial incubation for 10 min at $95^{\circ} \mathrm{C}$, followed by 35 cycles with the following profile: $1 \mathrm{~min}$ at $94^{\circ} \mathrm{C}, 1 \mathrm{~min}$ at $56^{\circ} \mathrm{C}$, and $1 \mathrm{~min}$ at $72^{\circ} \mathrm{C}$ using the Mastercycler Personal Thermocycler (Eppendorf Scientific, Westbury, NY, USA). An aliquot of the reaction mixture was analyzed on a $1.5 \%$ agarose gel, stained with ethidium bromide and the ultraviolet (UV)-illuminated image was captured digitally with a CCD camera (UVP, Upland, CA, USA).

\section{Western analysis}

All reagents were purchased from Invitrogen, unless stated otherwise. HGF, HDP, WS1, and FSF cell cultures were lysed in Radio-Immunoprecipitation Assay (RIPA) buffer, and $10 \mu \mathrm{g}$ of protein lysate was loaded onto $10 \%$ Bis-Tris gel (for SCF) or 3-8\% Tris-acetate (for c-kit). The gels were transferred to polyvinylidene difluoride (PVDF) membranes, which were incubated with goat polyclonal antibody raised against human SCF (R \& D Systems, Minneapolis, MN, USA), diluted 1:500, followed by a horseradish peroxidase-labeled secondary antibody diluted $1: 10,000$ (CalBiochem-Novabiochem, San Diego, CA, USA). The primary anti-c-kit antibody (Santa Cruz Biotechnology, Santa Cruz, CA, USA) was diluted $1: 700$, followed by application of a secondary antibody (CalBiochem-Novabiochem), diluted $1: 10,000$. Antibody binding was detected using a commercially available chemiluminescent system (Roche Diagnostics, Indianapolis, IN, USA) and the signal was captured and digitized with a CCD camera (UVP). For the blocking study, recombinant human SCF (R \& D Systems) was incubated with anti-SCF immunoglobulin at fivefold excess. The blocking peptide of c-kit (Santa Cruz Biotechnology) was incubated with anti-c-kit immunoglobulin at seven-fold excess. 


\section{Flow cytometry}

All fluorochrome-conjugated antibodies were purchased from BD Biosciences (Rockville, MD, USA). Anti-SCF immunoglobulin and isotype control for SCF were purchased from R \& D Systems. HDP or HGF cells were trypsinized and quickly resuspended at a concentration of $3 \times 10^{6}-10^{7}$ cells $\mathrm{ml}^{-1}$ in phosphate-buffered saline (PBS) containing 5\% FBS; $100 \mu \mathrm{l}$ aliquots were used for antibody incubations. For SCF detection, cells were incubated with an antihuman SCF monoclonal antibody (30 $\mu$ l per $10^{6}$ cells), followed by an antimouse $\operatorname{IgG}$ fluorescein isothiocyanate (FITC)-conjugated secondary antibody $\left(10 \mu \mathrm{l}\right.$ per $10^{6}$ cells). For c-kit, cells were incubated with an allophycocyanin (APC)-conjugated antihuman c-kit monoclonal antibody $\left(30 \mu \mathrm{l} / 10^{6}\right.$ cells $)$. In order to identify any cells that co-expressed the receptor and ligand, sequential incubations of the same sample with all three antibodies (anti-SCF, FITC-conjugated anti-IgG, APCconjugated anti-c-kit) were performed. Non-specific binding was assessed through the use of isotype-negative controls, added at the same concentrations. Fluorescence was measured using a FACS Aria three-laser cell sorter (BD Biosciences, San Jose, CA, USA). Approximately $10^{5}$ cells were measured from each sample. Experiments were repeated at least three times for each cell type and data from one representative experiment are presented. In order to provide comparable results between experiments, a threshold of fluorescence intensity was established in each experiment, so that $\geq 97 \%$ of cells in the unstained and/or isotype-control groups were below this threshold. In HGF cells, however, the threshold for FITC fluorescence (antiSCF) was set at $\geq 94 \%$ owing to autofluorescence of these cells at the respective wavelengths. This background fluorescence was subtracted from the experimental value to yield the true percentage of SCF- and/or c-kit-positive cells. As, in most cases, fluorescence for unstained cells was slightly higher than that for the isotype controls, unstained values were used as background.

\section{ELISA}

sSCF from HGF and HDP supernatants was quantified using a commercially available ELISA kit (R \& D Systems). The absorbance $(A)$ was measured at $450 \mathrm{~nm}$ using a microplate reader (Dynex, Chantilly, VA, USA). The values were plotted against a standard curve of recombinant human SCF and normalized to cell number.

\section{Results}

\section{HGF and dental pulp cells express both SCF isoforms}

In order to explore whether HGF and HDP express SCF, and also to distinguish between the two SCF isoforms, RT-PCR was performed using a set of primers flanking exon 6. Both $\mathrm{HGF}$ and $\mathrm{HDP}$ cells showed a strongly staining band at $416 \mathrm{bp}$ (sSCF) and a weaker band at $330 \mathrm{bp}(\mathrm{mSCF})$, indicating expression of the two alternatively spliced transcripts (Fig. 2). The difference in intensity between the two bands has been previously reported and probably reflects a difference in amplification efficacy of the two transcripts by the same primer set (19).

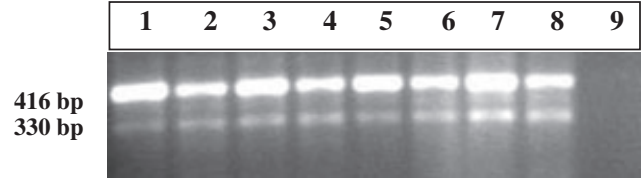

Fig. 2. Reverse transcription-polymerase chain reaction (RTPCR) exhibiting expression of the exon 6(+) transcript $(416 \mathrm{bp})$ and the exon $6(-)$ transcript $(330 \mathrm{bp}$ ) in human dental pulp (HDP) (lanes 1-4) and human gingival fibroblast (HGF) (lanes 5-8) cells. Each lane represents a separate experiment. Lane 9 is the negative control double distilled water $\left[\mathrm{ddH}_{2} \mathrm{O}\right]$.

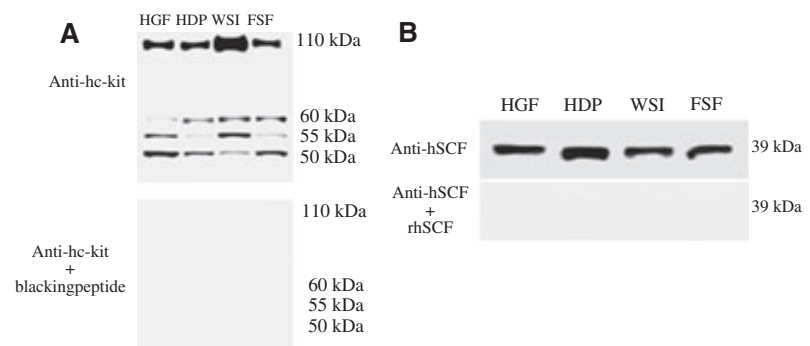

Fig. 3. Western analysis of c-kit (A) and stem cell factor (SCF) (B) in human gingival fibroblast (HGF), human dental pulp (HDP), human skin fibroblast (WS1), and human foreskin fibroblast (FSF) cells. (A) Top panel: primary immunoreactivity against the anti-c-kit immunoglobulin can be seen at $110 \mathrm{kDa}$ in all cell types, which is the expected molecular weight (MW) of the intact receptor. Secondary bands are detected at lower MWs, reflecting fragments of the internalized receptor. Bottom panel: pre-incubation of the antibody with its blocking peptide abolishes all bands, indicating specificity of the reaction. (B) SCF immunoreactivity is detected at $39 \mathrm{kDa}$ in all cell types, representing the heavily glycosylated transmembrane isoform (top panel). Pre-incubation of the anti-SCF immunoglobulin with an excess of human soluble SCF abolishes the signal (bottom panel).

\section{The two cell types express SCF and c-kit}

Western analysis revealed that both cell types express membrane-associated SCF as well as c-kit (Fig. 3). SCF immunoreactivity was detected at $39 \mathrm{kDa}$, which is consistent with other reports and reflects the molecular weight of the glycosylated monomer. Incubation of the anti-SCF immunoglobulin with human SCF prior to membrane incubation abolished the signal, indicating specificity of the antibody (Fig. 3A). When an antibody specific for c-kit was used, a primary band at $110 \mathrm{kDa}$ was clearly identifiable, representing the full-length receptor chain (Fig. 3B). However, c-kit immunoreactivity was also detected at lower molecular weights (50, 55 , and $60 \mathrm{kDa}$ ). Similar bands have been reported in the literature and probably represent fragments of the internalized receptor (7). This was further confirmed when anti-c-kit immunoreactivity was blocked by preincubating the antibody with its immunogen, resulting in elimination of all bands (Fig. 3B).

\section{Co-expression of the receptor and its ligand is present in a subset of either cell type}

Because the HDP and HGF cultures are heterogeneous, it is possible that SCF and c-kit are expressed in different 
subsets. To explore the potential co-expression of the ligand and receptor, we performed FACS analysis using monoclonal antibodies specific to SCF or c-kit. In both HDP and HGF cells, three distinct subpopulations were identified based on antibody reactivity (Fig. 4A,B) those expressing SCF alone, those expressing c-kit alone, and those co-expressing the ligand and the receptor. Under our culture conditions, the percentage of overall SCF-positive cells was higher in HDP compared with HGF (16.7\% vs. $5.1 \%$; panel I), whereas c-kit expression was similar (19.2\% vs. $20.4 \%$; panel II). Most importantly, $\approx 10.3 \%$ of HDP cells and $5.6 \%$ of HGF cells co-expressed SCF and c-kit (panels III and IV). It should be noted, however, that HDP cells from different donors showed considerable variability. We tested five different primary cultures, where the double-positive populations ranged from 2 to $15 \%$. Similarly, the ranges for SCF alone and c-kit alone positive cells were $5-20 \%$ and 10 $40 \%$, respectively. HGF cells come from a single source, so the values from repeated experiments were more consistent.

\section{Both cell types secrete soluble SCF in culture supernatants}

To elucidate in greater detail the potential of HGF and HDP cells to secrete SCF, we analyzed conditioned media, using a commercially available ELISA, over a period of $2 \mathrm{wk}$. Although both cell types secreted measurable amounts of SSCF, HDP cells were much stronger producers than HGF cells, even when sSCF values were normalized to cell number (Fig. 5A). Interestingly, HDP cells also had a much higher proliferative capacity (Fig. 5B). Furthermore, sSCF production followed a different pattern in the two cell types: in HGF cells, sSCF in culture supernatants increased in a linear pattern over the culture period, whereas in HDP cells, sSCF showed a steeper increase, peaking midway through the culture (day 11) and dropping sharply afterwards. Day 11 seemed to be a key transitional point in the HDP culture, after which the rate of proliferation slowed down and alkaline phosphatase (ALP) activity increased considerably, suggestive of osteogenic differentiation under these culture conditions (Fig. 5B). In HGF cells, ALP activity was practically negligible.

\section{Discussion}

The SCF/c-kit system plays a role in hematopoesis, gametogenesis, and melanogenesis by influencing survival, proliferation, and differentiation of stem cells (2). In most developmental models, the microenvironment contains separate c-kit $(+)$ and $\operatorname{SCF}(+)$ cells, whereas co-expression of these two molecules has been linked to neoplastic changes (14). Although co-expression of SCF and c-kit has been reported in non-neoplastic cells $(9,11)$, no functional roles for this co-expression have been addressed. In the present report we establish, for the first time, the presence of both SCF and c-kit in mesenchymal cells of the oral cavity and provide evidence of a possible
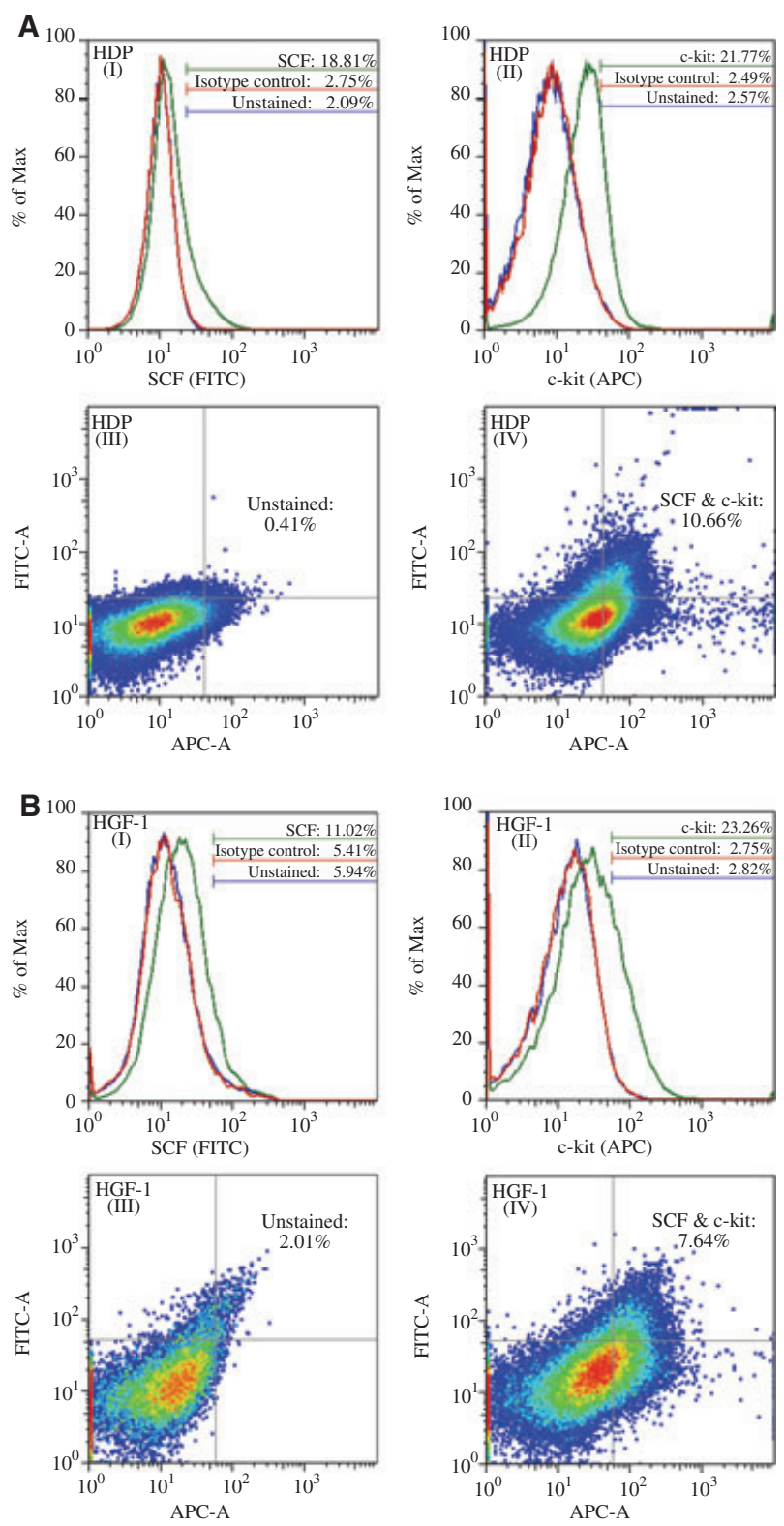

Fig. 4. Fluorescence-activated cell sorter (FACS) analysis of human dental pulp (HDP) (A) and human gingival fibroblast-1 (HGF-1) (B) cells for stem cell factor (SCF) and c-kit expression (panels I-IV). (I) Cells unstained, incubated with anti-SCF immunoglobulin alone or its isotype control; parameters set for fluorescein isothiocyanate (FITC) fluorescence. (II) Cells unstained, incubated with anti-c-kit immunoglobulin alone or its isotype control; parameters set for allophycocyanin (APC) fluorescence. (III) Unstained cells analyzed for FITC and APC fluorescence. (IV) Cells sequentially incubated with anti-SCF and anti-c-kit immunoglobulin and analyzed for FITC and APC fluorescence. The threshold for background fluorescence was set so that $\geq 97 \%$ of cells in the unstained or isotype-control groups were negative $(\geq 94 \%$ for anti-SCF binding in HGF cells owing to autofluorescence). Because, in most cases, the unstained background values were slightly higher than those for the isotype controls, the true percentage of positive cells was calculated by subtracting the unstained group value from the antibody group value. 

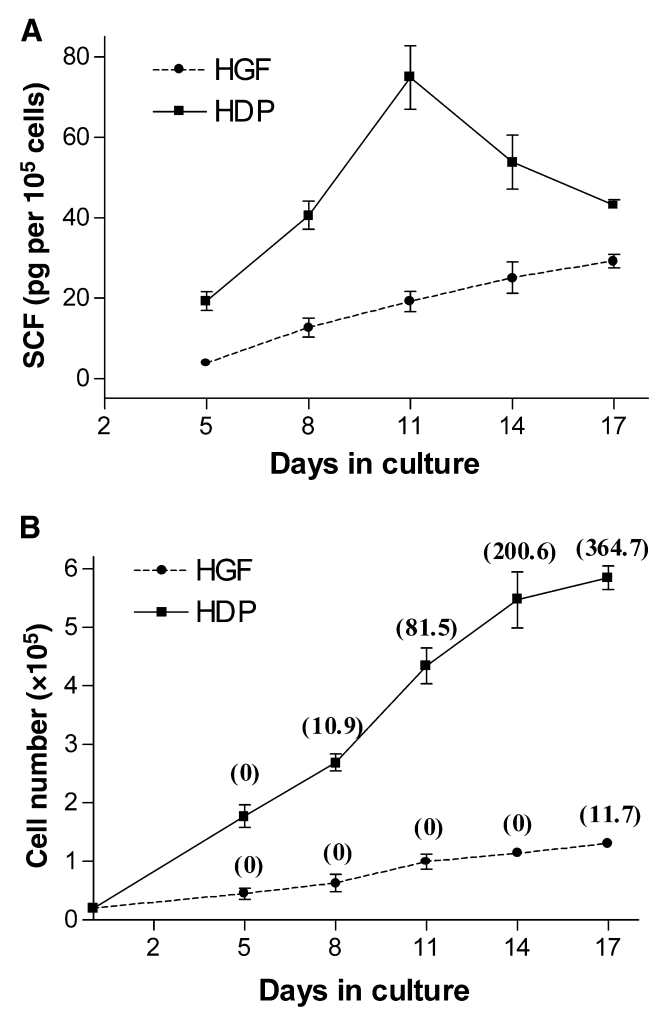

Fig. 5. Soluble stem cell factor (SCF) release in culture supernatants (A) and proliferation patterns (B) of human dental pulp (HDP) and human gingival fibroblast (HGF) cells. (A) Soluble SCF, which had accumulated in culture supernatants over a 3-d period, was measured using an enzyme-linked immunosorbent assay (ELISA) and normalized to cell number. (B) Cell number of HDP and HGF cultures. Numbers in parentheses indicate alkaline phosphatase activity at the specific time points normalized to total protein (pmoles paranitrophenol $\mathrm{min}^{-1}$ per $\mathrm{mg}$ of protein). Bars in (A) and (B) reflect the standard deviation of triplicate wells.

association between SCF production and cell differentiation or aging in vitro.

Expression of SCF in cells of mesenchymal origin has clearly been established in rodents (20) as well as in humans $(19,21)$. Human stromal cells constituvely express both SCF isoforms (21) and can release soluble SCF in the bone marrow microenvironment (2). Consistent with this pattern, we find that fibroblast-like cells from the gingiva and dental pulp express both exon $6(+)$ and exon 6(-) isoforms of SCF (Figs 2-4) and can produce soluble SCF (Fig. 5A). Although SCF expression at the mRNA level has been reported in gingival biopsies (22), as well as in buccal mucosa fibroblasts (16), no systematic analysis has been attempted. Given the complex regulatory mechanisms that govern SCF signaling, identification of the specific isoforms is of paramount importance. SSCF and $\mathrm{mSCF}$ activate different signaling pathways after binding to c-kit and may exert distinct biologic effects (5). The temporal pattern of SSCF production in long-term cultures showed some interesting trends (Fig. 5A). HDP cells appeared to be more robust producers of SCF compared with gingival fibroblasts. This difference could be associated with a higher proliferative activity and/or a tendency for differentiation along the osteoblastic pathway, as indicated by the significantly elevated levels of ALP activity (Fig. 5B). This is consistent with the reported increase of SCF mRNA during osteogenic differentiation of mouse bone marrow stromal cells (23). Alternatively, SCF might serve as a survival factor and its increase could reflect cell aging in culture, because it has been reported that fibroblasts from older individuals release more SCF than their counterparts from younger subjects (24). Moreover, HDP cells showed a peak by day 11 and a sharp drop afterwards, whereas HGF cells showed a gradual increase in SCF levels throughout the culture period. As HDP cells have the capacity for multilineage differentiation (25), the bimodal pattern of sSCF production could reflect a phenotypic transition of the cells in culture, which cannot be monitored by proliferation or osteogenic markers.

Although c-kit expression is often associated with cells of neuroectodermal origin (2), its presence in cells of mesenchymal origin is equivocal. Mouse fibroblasts are considered to lack c-kit (14), a characteristic also reported for bone marrow stromal cells from mouse (26) and human (19). In contrast, c-kit expression has been reported in mouse mesenchymal progenitor cells (27) and activated human mesenchymal stem cells (28). Furthermore, a subpopulation of murine mesenchymal stem cells, capable of differentiation along the fibrogenic, osteogenic, chondrogenic or adipogenic pathways, responded to exogenous SCF stimulation with increased proliferation (29). In a recent report, LAINO et al. isolated a c-kit ${ }^{+} / \mathrm{CD} 35^{+} / \mathrm{CD} 45^{-}$cell population from human adult dental pulp that can produce a bone-like tissue in vitro (15). The presence of c-kit in dental pulp cells could be attributed to the ectomesenchymal origin of these cells (15). Originating from human pulp tissue without any subcloning, these cultures are heterogeneous, but the cell origin is clearly mesenchymal with no evidence of hematopoietic or ectodermal contamination (17). It is possible that pulp cells with pericytic or myofibroblastic characteristics could account for c-kit expression, as has been reported in other tissues (30). The developmental origin of HGF cells could also account for their c-kit expression. However, our two fibroblast control cell lines (WS1 and FSF) also expressed c-kit (Fig. 3), thus supporting the inference that c-kit expression in human cell types is more widespread, compared with mouse.

The most striking finding is the presence of distinct subpopulations in both HDP and HGF that co-express the ligand and its receptor (Fig 4A,B). Co-expression of SCF and c-kit has been reported in osteosarcoma cells (31), where an autocrine loop promoting cell proliferation was proposed. Our cells do not show evidence of any neoplastic transformation, therefore the possible role of any autocrine signaling requires further study. Interestingly, HDP cultures had a higher proportion of SCFpositive cells than the HGF cultures (Fig. 4A,B, panel I), which corroborated the ELISA findings (Fig. 5A). The flow cytometry results for SCF should be interpreted with caution, because the epitope recognized by the antiSCF immunoglobulin is found in the cleavable part of 
the molecule. Therefore, it is possible that cells containing the exon $6(+)$ isoform could have been counted as negative if sSCF had already been cleaved at the time the assay was performed. Our experimental design does not allow distinction of exon $6(+)$ and exon 6(-) cells. Finally, the variability in SCF and c-kit reactivity between cultures from different subjects is an expected finding. In addition to donor variability, expression of $\mathrm{SCF}$ and/or c-kit might be influenced by the proliferation and/or differentiation status of the individual cultures.

Given the potential autocrine or paracrine activity of $\mathrm{SCF}$, the functional role of c-kit in pulp and gingival fibroblastic cells becomes a point of interest. Signaling through c-kit is known to be very important for cell survival and differentiation; however, cleavage of the plasma membrane-associated receptor by proteases releases a soluble fraction, which has the ability to bind SCF and modulate its bioactivity (8). This seems to be the case with human endothelial cells, which express a cleavable, high-affinity c-kit receptor that apparently fails to respond to exogenous SCF stimulation (32). Co-expression and possible cleavage of SCF and c-kit can result in a complex autocrine/ paracrine regulatory system, which merits further exploration.

In summary, this is the first systematic report in which SCF and c-kit expression is studied at the transcriptional, translational, and post-translational level in cells of mesenchymal origin. Furthermore, we provide evidence implicating a possible role for SCF and c-kit in oral tissue homeostasis. Future studies will aim to explore the mechanism of action of the SCF/c-kit pathway in these cells.

Acknowledgements - The research was supported by Institutional funds to Eleni Gagari and Petros D. Damoulis, as well as by a grant from the American Association of Endodontists to Eleni Gagari, a grant from the March of Dimes Foundation to Heleni Vastardis, and grants from the USAMRC-DOD (DAMD-17-03-1-0607; BC050864), NIH (R01-AR48799, R01DE13360), and the St Giles Foundation to P. V. Hauschka.

\section{References}

1. Williams De, Eisenman J, Baird A, Rauch C, Van Ness K, March CJ, Park lS, Martin U, Mochizuki DY, Boswell HS. Identification of a ligand for the c-kit proto-oncogene. Cell 1990; 63: 167-174.

2. BRoudy VC. Stem cell factor and hematopoiesis. Blood 1997; 90: $1345-1364$.

3. Longley BJ, Tyrrell L, Ma Y, Williams DA, Halaban R, Langley K, Lu HS, Schechter NM. Chymase cleavage of stem cell factor yields a bioactive, soluble product. Proc Natl Acad Sci USA 1997; 94: 9017-9021.

4. Arakawa T, Yphantis Da, Lary JW, Narhi lo, Lu hS, Prestrelski SJ, Clogston CL, Zsebo KM, Mendiaz EA, WYPYCH J. Glycosylated and unglycosylated recombinant-derived human stem cell factors are dimeric and have extensive regular secondary structure. J Biol Chem 1991; 266: 1894218948.

5. Trieselmann NZ, Soboloff J, Berger SA. Mast cells stimulated by membrane-bound, but not soluble, steel factor are dependent on phospholipase C activation. Cell Mol Life Sci 2003; 60: 759-766.
6. Caruana G, Cambareri AC, Ashman LK. Isoforms of c-Kit differ in activation of signalling pathways and transformation of NIH3T3 fibroblasts. Oncogene 1999; 18: 5573-5581.

7. Levesque JP, Hendy J, Winkler IG, Takamatsu Y, Simmons PJ. Granulocyte colony-stimulating factor induces the release in the bone marrow of proteases that cleave c-KIT receptor (CD117) from the surface of hematopoietic progenitor cells. Exp Hematol 2003; 31: 109-117.

8. Dahlen DD, Lin NL, Liu YC, Broudy VC. Soluble Kit receptor blocks stem cell factor bioactivity in vitro. Leuk Res 2001; 25: 413-421.

9. Welker P, Grabbe J, Gibbs B, Zuberbier T, Henz BM. Human mast cells produce and differentially express both soluble and membrane-bound stem cell factor. Scand J Immunol 1999; 49: 495-500.

10. Doneda L, Klinger FG, Larizza L, De Felici M. KL/Kit co-expression in mouse fetal oocytes. Int J Dev Biol 2002; 46: 1015-1021.

11. Welker P, Schadendorf D, Artuc M, Grabbe J, Henz BM. Expression of SCF splice variants in human melanocytes and melanoma cell lines: potential prognostic implications. $\mathrm{Br} J$ Cancer 2000; 82: 1453-1458.

12. Zheng R, Klang K, Gorin NC, Small D. Lack of KIT or FMS internal tandem duplications but co-expression with ligands in AML. Leuk Res 2004; 28: 121-126.

13. Hines SJ, Organ C, Kornstein MJ, Krystal GW. Coexpression of the c-kit and stem cell factor genes in breast carcinomas. Cell Growth Differ 1995; 6: 769-779.

14. Caruana G, Cambareri aC, Gonda TJ, ashman LK. Transformation of NIH3T3 fibroblasts by the c-Kit receptor tyrosine kinase: effect of receptor density and ligand-requirement. Oncogene 1998; 16: 179-190.

15. Laino G, d'Aquino R, Graziano A, Lanza V, Carinci F, Naro F, Pirozzi G, Papaccio G. A new population of human adult dental pulp stem cells: a useful source of living autologous fibrous bone tissue (LAB). J Bone Miner Res 2005; 20: 1394-1402.

16. Okazaki M, Yoshimura K, Uchida G, Hari K. Elevated expression of hepatocyte and keratinocyte growth factor in cultured buccal-mucosa-derived fibroblasts compared with normal-skin-derived fibroblasts. J Dermatol Sci 2002; 30: 108115.

17. Gronthos S, Mankani M, Brahim J, Robey PG, Shi S. Postnatal human dental pulp stem cells (DPSCs) in vitro and in vivo. Proc Natl Acad Sci USA 2000; 97: 13625-13630.

18. Asahina I, Sampath TK, Hauschka PV. Human osteogenic protein-1 induces chondroblastic, osteoblastic, and/or adipocytic differentiation of clonal murine target cells. Exp Cell Res 1996; 222: 38-47.

19. Aye mt, hashemi S, Leclair B, Zeibdawi A, Trudel E, Halpenny M, Fuller V, Cheng G. Expression of stem cell factor and c-kit mRNA in cultured endothelial cells, monocytes and cloned human bone marrow stromal cells (CFU-RF). Exp Hematol 1992; 20: 523-527.

20. Anderson DM, Lyman SD, Baird A, Wignall JM, Eisenman J, Rauch C, March CJ, Boswell HS, Gimpel SD, Cosman D, WiLliams DE. Molecular cloning of mast cell growth factor, a hematopoietin that is active in both membrane bound and soluble forms. Cell 1990; 63: 235-243.

21. Heinrich MC, Dooley DC, Freed AC, Band L, Hoatlin Me, Keeble WW, Peters St, Silvey KV, Ey FS, Kabat D. Constitutive expression of steel factor gene by human stromal cells. Blood 1993; 82: 771-783.

22. Schierano G, Bassi F, Gassino G, Mareschi K, Bellone G, PRETI G. Cytokine production and bone remodeling in patients wearing overdentures on oral implants. $J$ Dent Res 2000; 79: $1675-1682$.

23. Yanai N, Obinata M. Oncostatin m regulates mesenchymal cell differentiation and enhances hematopoietic supportive activity of bone marrow stromal cell lines. In Vitro Cell Dev Biol Anim 2001; 37: 698-704.

24. Imokawa G, Yada Y, Morisaki N, Kimura M. Biological characterization of human fibroblast-derived mitogenic factors for human melanocytes. Biochem J 1998; 330: 1235-1239. 
25. Gronthos S, Brahim J, Li W, Fisher LW, Cherman N, Boyde A, Denbesten P, Robey PG, Shi S. Stem cell properties of human dental pulp stem cells. J Dent Res 2002; 81: 531-535.

26. Baddoo M, Hill K, Wilkinson R, Gaupp D, Hughes C, Kopen GC, Phinney DG. Characterization of mesenchymal stem cells isolated from murine bone marrow by negative selection. J Cell Biochem 2003; 89: 1235-1249.

27. Sun S, Guo Z, Xiao X, Liu B, Liu X, Tang PH, Mao N. Isolation of mouse marrow mesenchymal progenitors by a novel and reliable method. Stem Cells 2003; 21: 527-535.

28. Huss R, Moosmann S. The co-expression of CD117 (c-kit) and osteocalcin in activated bone marrow stem cells in different diseases. Br J Haematol 2002; 118: 305-312.

29. Hall Fl, Han B, Kundu RK, Yee A, Nimni ME, Gordon EM. Phenotypic differentiation of TGF-beta1-responsive pluripotent premesenchymal prehematopoietic progenitor $(\mathrm{P} 4$ stem) cells from murine bone marrow. J Hematother Stem Cell Res 2001; 10: 261-271.

30. Powell DW, Mifflin RC, Valentich JD, Crowe SE, Saada JI, WeSt AB. Myofibroblasts. II. Intestinal subepithelial myofibroblasts. Am J Physiol 1999; 277: C183-C201.

31. Hitora T, Yamamoto T, Akisue T, Marui T, Nakatani T, Kawamoto T, Nagira K, Yoshiya S, Kurosaka M. Establishment and characterization of a KIT-positive and stem cell factor-producing cell line, KTHOS, derived from human osteosarcoma. Pathol Int 2005; 55: 41-47.

32. Broudy VC, Kovach NL, Bennett LG, Lin N, Jacobsen FW, KIDD PG. Human umbilical vein endothelial cells display highaffinity $c$-kit receptors and produce a soluble form of the $c$-kit receptor. Blood 1994; 8: 2145-2152. 